\title{
A STUDY OF A BISTEREOGENIC MESOGEN FOR THE DEVELOPMENT OF ORTHOCONIC ANTIFERROELECTRIC LIQUID CRYSTAL MATERIALS
}

\author{
JAN LAGERWALL ${ }^{\mathrm{a}}$, CHRIS YATES $^{\mathrm{b}}$, SEBASTIAN RAUCH $^{\mathrm{b}}$, \\ GERD HEPPKE ${ }^{b}$ \\ ${ }^{a}$ University of Stuttgart, Institute of Physical Chemistry, 70569 Stuttgart, \\ Germany; ${ }^{\mathrm{b}}$ Technical University Berlin, Department of Chemistry, \\ 10623 Berlin, Germany.
}

The first orthoconic antiferroelectric liquid crystals (OAFLCs), i.e. smectics where the optical director changes direction by $90^{\circ}$ between neighboring layers, were mixtures of partially fluorinated monostereogenic compounds. They have successfully demonstrated the orthoconic properties (orientation-independent dark state between crossed polarizers) but suffer from too high polarization and too short helical pitch, necessitating very thin samples. Using an $(S, R)$ bistereogenic OAFLC we have obtained the orientation-independent dark state in rather thick samples, but several other problems arise with this compound. The strongly first-order $\mathrm{SmA}^{*}-\mathrm{SmC}_{\mathrm{a}}$ * transition produces defects leading to light leakage. In order to be switchable the sample must furthermore be mixed with chiral dopants, generally reducing the tilt angle as well as a shortening the helical pitch. Finally, a SmC* phase often appears in the phase sequence of the mixture, strongly dominating over the desired $\mathrm{SmC}_{\mathrm{a}}{ }^{*}$ phase in thin cells.

Keywords orthoconic antiferroelectric liquid crystals; high optical tilt; defect-generated light scattering; chiral doping; confinement effects; supercooling 


\section{A BISTEREOGENIC ORTHOCONIC AFLC}

\section{INTRODUCTION}

It was recently realized that if, in an antiferroelectric liquid crystal (AFLC) material, the principal axes of the dielectric tensors corresponding to alternate smectic layers are perpendicular, such a material becomes optically uniaxial in its surface-stabilized state, with the optic axis perpendicular to the cell plate [1-3]. Hence such materials optically correspond to a nematic in its homeotropic state, giving extinction between crossed polarizers regardless of how the sample is rotated. AFLC materials with these properties are referred to as orthoconic AFLCs or OAFLCs. They evidently give new hope for solving the long standing dark state problem in AFLC devices. However, the desired orientation-independent dark state will arise only if a number of requirements are fulfilled by the materials used as well as by the type of alignment obtained in the sample cell or device.

The AFLC material has to have an optical director tilt $\theta$ of $45^{\circ}$ (or close to $45^{\circ}$ ) in the anticlinic state, i.e. in the $\mathrm{SmC}_{\mathrm{a}}{ }^{*}$ phase in the absence of fields. There is no straight-forward way to measure $\theta$ in an anticlinic system, so the standard experimental procedure is to switch the sample with an electric field to the synclinic state, in which $\theta$ can easily be measured. One then assumes that this 'field-induced apparent tilt' reflects the tilt also in the anticlinic state. For ordinary rigid rod-like molecular structures this assumption is likely to be valid in most cases, but there are cases where the synclinic and anticlinic tilt are not necessarily comparable, e.g. if the actual molecular shape can be expected to be affected by the field. Such a situation might arise for instance if the molecules possess flexible groups connecting rigid structures which can all be expected to respond to the field as well as contribute to the optical properties.

In the special case of OAFLCs it is actually possible to assess the optical director tilt in the anticlinic state, in the sense that one can test if

$\theta_{\text {anticlinic }} \approx 45^{\circ}$ or not. On a qualitative level this is easily done by rotating the sample between crossed polarizers and checking if the transmission is constantly very low. A more accurate, but also considerably more complex, method based on wave guiding properties has been proposed and tested by Scalia et al. [4]. The test in both cases however requires that the sample also fulfils the important special conditions of alignment.

As for the alignment, it has to guarantee a very high quality surfacestabilization such that the tilt plane is strictly parallel to the sample surface [5]. Good surface stabilization on the other hand sets another requirement on the AFLC material, that of a not too short helical pitch, 


\section{J. LAGERWALL ET AL.}

because for optimized electrooptic device efficiency the sample thickness should be close to that which gives the switched synclinic sample the function of a $\lambda / 2$ wave plate.

So far, the most successful OAFLC materials have been multi-component mixtures where the constituents all have biphenyl-ester-phenyl cores, a chiral aliphatic terminal chain and in the opposite end a partially fluorinated achiral chain [1]. These mixtures exhibit an Isotropic-SmC*$\mathrm{SmC}_{\mathrm{a}}{ }^{*}$-Crystal phase sequence. Their main deficiency has been that the helical pitch in the $\mathrm{SmC}_{\mathrm{a}}{ }^{*}$ phase is less than $1 \mu \mathrm{m}$, requiring inconveniently thin cells in order to assure surface stabilization. An alternative approach is to use bistereogenic AFLCs, where the molecules have one chiral carbon in each end chain. MHTAC, one of the first liquid crystals in which the anticlinic $\mathrm{SmC}_{\mathrm{a}}$ * phase was observed [6-8], is a bistereogenic molecule exhibiting a tilt $\theta \sim 45^{\circ}$ [9]. However, the compound has never been studied in the surface-stabilized state, hence its properties as an OAFLC were not discovered.

We have chosen to study a series of bistereogenic AFLCs closely related to the MHTAC molecule, but where the Schiff's base-containing core has been replaced by two ester-linked biphenyls. The homologue $(S, R)$ - M7BBM7 ( $c f$. Table 1) turned out to be promising as an OAFLC host since it exhibits a high-optical tilt $\mathrm{SmC}_{\mathrm{a}} *$ phase $\left(\theta \sim 45^{\circ}\right.$ at room temperature, to which the $\mathrm{SmC}_{\mathrm{a}}$ * phase can easily be supercooled) as well as a very long helical pitch, an effect of the presence of $(S)$ - and $(R)$ chiral carbons in the same molecule. The $(S, R)$ combination obviously has the drawback that the spontaneous polarization is close to zero, so to obtain a useful OAFLC one also needs to find a chiral dopant giving an increased polarization without affecting tilt, pitch and phase sequence too much. In this regard, a number of different dopants have been investigated. The optical properties of the anticlinic state have been studied in doped as well as undoped (S,R) - M7BBM7 samples, adding to the understanding of the benefits and problems of OAFLCs by giving information on how other high-tilt AFLC materials, fundamentally different from the mixtures used so far, may behave in a device configuration.

\section{EXPERIMENTAL}

The pure host material and the chirally doped mixtures were heated into the isotropic phase and filled into commercial EHC cells of $4 \mu \mathrm{m}$ thickness. The alignment layer was a polyimide coating supposed to give a 


\section{A BISTEREOGENIC ORTHOCONIC AFLC}

TABLE 1. Compounds used in the study, their phase sequences, optical director tilt $\theta$ a and spontaneous polarization $P_{s}$

\begin{tabular}{lll}
\hline $\begin{array}{l}\text { Compound name, molecule and phase sequence } \\
\left.\text { (transition temperatures in }{ }^{\circ} \mathrm{C}\right)\end{array}$ & $\begin{array}{l}\theta_{\max } \\
(\text { degrees })\end{array}$ & $\begin{array}{l}P_{S}(\max ) \\
(\mathrm{nCcm})\end{array}$ \\
\hline M7BBM7 & $\sim 45$ & $\begin{array}{l}(S, R) \& \\
(R, S): \sim 0^{\mathrm{c}}\end{array}$
\end{tabular}

$(S, R) \&(R, S):$ Cr. - 56- $\mathrm{SmC}_{\mathrm{a}}{ }^{*}-120-\mathrm{SmA}^{*}-135$ - Iso. ${ }^{\mathrm{b}}$

$(S, S) \&(R, R):$ Cr. - $57-\mathrm{SmC}_{\mathrm{a}}{ }^{*}-65-\mathrm{SmQ}^{*}-85$ - Iso.

$\begin{array}{lll}\text { M5BBM5 } & \sim 45 & \text { Not } \\ \text { measured }\end{array}$

$(S, S) \&(R, R):$ Cr. - 44 - $\mathrm{SmC}_{\mathrm{a}}{ }^{*}-111$ - Iso.



very small director tilt at the surface of the cell. The optical tilt angle in the synclinic state was measured using a technique first proposed by Bahr and Heppke where the optical transmission $(T)$ of both states during sat- 


\section{J. LAGERWALL ET AL.}

urated square wave switching was measured for several consecutive sample orientations $\varphi$. By fitting two $\sin ^{2}$ functions to the two resulting $T(\varphi)$ data sets the tilt angle can be extracted [10]. The spontaneous polarization was measured by integrating the polarization reversal current while switching the sample with a triangular wave [11].

\section{RESULTS AND DISCUSSION}

\section{The Undoped Surface-Stabilized Orthoconic AFLC Host}

The $(S, R)$ - M7BBM7 compound has an essentially non-helical structure, thus ought to be easily surface stabilized even in fairly thick cells. Since the compound is very close to being achiral (had the central ester linkage been replaced with a symmetric moiety there would have been a mirror plane perpendicular to the molecular long axis) it unfortunately has a very low spontaneous polarization. In order to switch the liquid crystal it therefore has to be chirally doped, rendering quantitative measurements of the optical tilt angle $\theta$ in the pure host material very difficult. Nevertheless, we can deduce from the texture photos shown in Figure 1 that $\theta_{\text {anticlinic }}$ in the $\mathrm{SmC}_{\mathrm{a}} *$ phase gets very close to $45^{\circ}$. At the transition from $\mathrm{SmA}^{*}$ - in which the sample had a bluish pink texture - to $\mathrm{SmC}_{\mathrm{a}}{ }^{*}$ a drastic decrease in birefringence $\Delta n$ took place as is seen through the sudden disappearance of color in the texture (lower row, first two pictures). On further cooling $\Delta n$ continuously decreased, reflecting an increasing tilt angle in an anticlinic structure, and at room temperature it was so low that the sample was dark grey even in the maximum transmission orientation. This shows that the conditions for OAFLC behavior are essentially fulfilled, i.e. $\theta_{\text {anticlinic }} \sim 45^{\circ}$ and the tilt plane is close to parallel to the cell substrates. (The fact that the defect-free regions in the lower right-hand picture of Figure 1 are dark grey rather than black may give the false impression that the sample is far from the orthoconic condition. However, the cell gap is $4 \mu \mathrm{m}$ - five to six times the sample thickness that has been used in earlier OAFLC studies - allowing some light transmission even if the birefringence is very close to zero.)

However, many defects were formed at the strongly first-order $\mathrm{SmA}^{*}-\mathrm{SmC}_{\mathrm{a}}{ }^{*}$ transition and these gave rise to considerable light scattering, thereby severely degrading the dark state. An important question is what the exact origin of the defects is. It is not the fact that the transition is direct $\mathrm{SmA}^{*}{ }_{-} \mathrm{SmC}_{\mathrm{a}}{ }^{*}$, much less common than $\mathrm{SmA}^{*}-\mathrm{SmC}^{*}$ or $\mathrm{SmA}^{*}$ $\mathrm{SmC}_{\alpha} *$ transitions, because we know of at least three examples where 


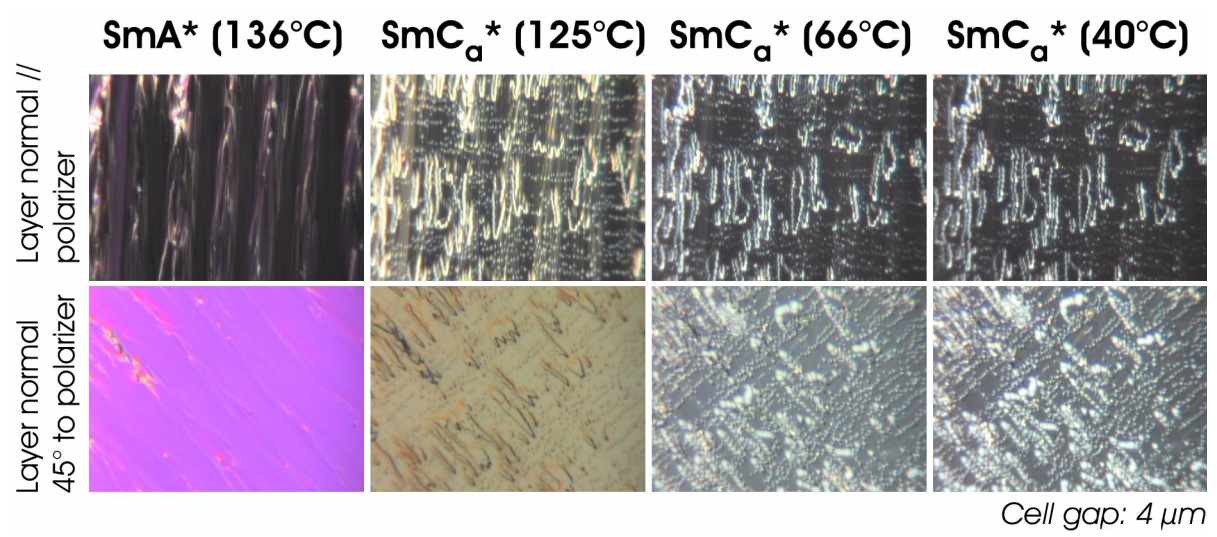

FIGURE 1. Textures observed on cooling the $(S, R)$ - M7BBM7 host. In the upper row the layer normal is aligned with the polarizer, giving minimum optical transmission, in the lower row it is rotated for maximum transmission. Note the drastic decrease in birefringence at the $\mathrm{SmA}^{*}-\mathrm{SmC}_{\mathrm{a}}$ * transition (texture changes from pink to colorless), and the very low maximum transmission in the $\mathrm{SmC}_{\mathrm{a}}{ }^{*}$ state reached close to room temperature (right picture, lower row). However, the many defects formed at the first-order tilting transition lead to some light leakage also at this temperature. The brightness of different pictures is not necessarily directly comparable due to the automatic exposure adjustment of the camera.

this transition introduces no defects at all. (In fact, in these materials EHPOCBC, MHP7BC and a partially fluorinated AFLC mixture - the changes to the texture induced by the transition are so small that it is difficult to detect the phase transition by means of texture observations alone.) But in contrast to $(S, R)-\mathrm{M} 7 \mathrm{BBM} 7$ the $\mathrm{SmA}^{*}-\mathrm{SmC}_{\mathrm{a}}{ }^{*}$ transition is in these cases second-order, and we therefore believe that the defects are related more to the order of the tilting transition than to which particular tilted phase follows the $\mathrm{SmA}^{*}$ phase on cooling. However, the OAFLC mixtures that have been used in earlier studies are not known to suffer from defects of this kind, despite the $\mathrm{SmA}^{*}-\mathrm{SmC} *$ transition being strongly first-order, so several parameters must play a role in how the texture responds to the transition. In the case of these mixtures, it is very likely that the fluorinated achiral terminal chain is of vital importance in maintaining a defect-free texture, because fluorinated smectics are known to exhibit tilting transitions with surprisingly little effect on layer spacing and thus with a minimum of defect generation, also in the case of AFLCs $[12,13]$. 


\section{J. LAGERWALL ET AL.}

\section{The chirally doped system}

Several chiral dopants were tested in order to produce a switchable mixture, $c f$. Table 1 . Doping with compounds that are structurally very similar to the $(S, R)$ - M7BBM7 host, such as $(S, S)$ - M7BBM7 and $(S, S)$ M5BBM5, was not successful due to the very poor alignment that these mixtures exhibited in our sample cells. When using more ordinary AFLC molecules with high spontaneous polarization as dopants, such as $(S)$ TFMHPOBC and $(S)$ - TFMHPBC - 11, the generation of a non-zero $P_{s}$ value was unfortunately coupled to a strong decrease in $\theta$. In addition, the amount of dopant needed to generate a polarization that allowed switching in all cases also decreased the helical pitch so much that surface stabilization was difficult to obtain in cells of reasonable thickness.

The most promising chiral dopant found was the $(S, R)$ - M7OBBL4 molecule. This molecule has the same core as the host but different terminal chains, making the molecule less symmetric and hence giving also the $(S, R)$ molecule a non-vanishing spontaneous polarization. In Figure 2, $\theta$ and $P_{s}$ of a $67 \%$ host $+37 \%$ dopant (by weight) mixture are displayed as a function of temperature. This mixture was quite useful since it had a tilt not far from $45^{\circ}$ and at the same time a polarization allowing switching into the synclinic state. However, the mixture had a relatively short pitch and it also exhibited a broad SmC* phase, a combination which turned out to be quite unfortunate. The short pitch calls for thin cell gap but in thin cells the $\mathrm{SmC}^{*}$ phase often has a strong tendency to supercool and in the worst case even expel the $\mathrm{SmC}_{\mathrm{a}}{ }^{*}$ phase in AFLCs [14]. In a

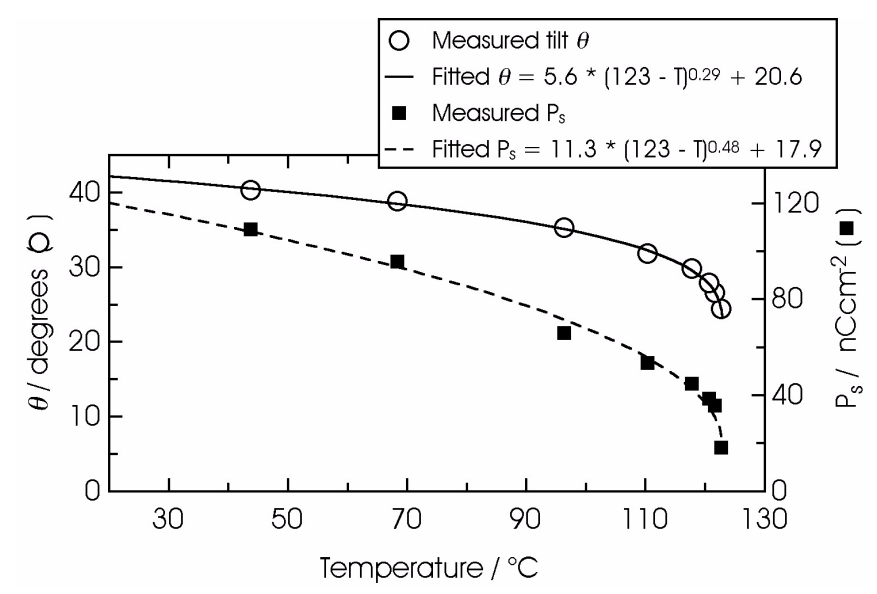

FIGURE 2. Optical tilt angle $\theta$ and spontaneous polarization $P_{S}$ of the $67 \%(S, R)-$ M7BBM7 + 37\% $(S, R)$ - M7OBBL4 mixture, together with best fits to each data series. 

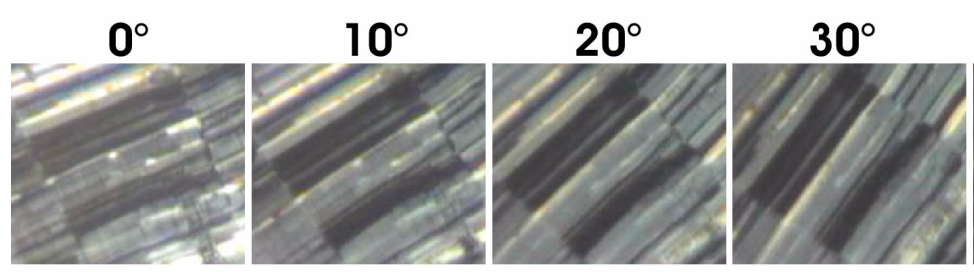

$40^{\circ}$

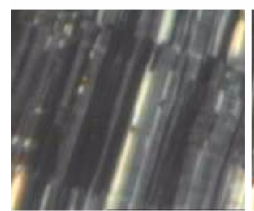

$50^{\circ}$

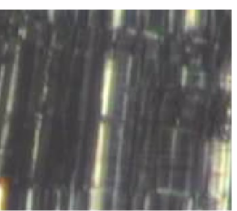

$60^{\circ}$

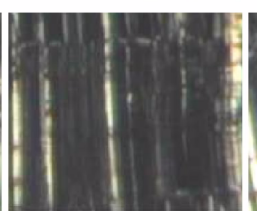

$70^{\circ}$

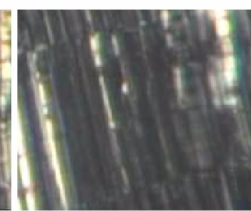

$80^{\circ}$
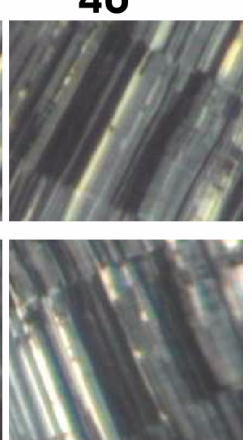

$90^{\circ}$

FIGURE 3. Two of the few well-aligned orthoconic domains of the chirally doped sample can be observed on either side of the center in each picture. As the sample is rotated by $90^{\circ}$ between crossed polarizers the transmission is here - locally - at a constant and very low level. The surrounding regions, not horizontally surface-stabilized, show a considerable variation in transmission as the sample is turned.

$1.5 \mu \mathrm{m}$ thick sample the mixture was on the verge of being surface stabilized, hence this cell represented the upper limit of useful cell gap. But the $\mathrm{SmC}_{\mathrm{a}}$ * phase actually never formed inside the electrode area of this cell; the SmC* phase was supercooled to room temperature. Outside the electrode area small parts of the sample did form the $\mathrm{SmC}_{\mathrm{a}}{ }^{*}$ phase. The picture series in Figure 3 shows two such small regions viewed at room temperature as the sample was rotated by 90 degrees. It is clear from their almost constantly black appearance that the mixture in the $1.5 \mu \mathrm{m}$ cell for practical purposes fulfills the OAFLC conditions (NB: the domains referred to were birefringent when heated to the $\mathrm{SmA}^{*}$ phase, i.e. the alignment was not homeotropic), albeit only in small portions of the cell, outside the area where an electric field can be applied. Hence, this mixture is mainly of academic interest, although a more useful mixture may be obtained by reducing the amount of chiral dopant.

\section{CONCLUSIONS AND OUTLOOK}

Orthoconic antiferroelectric liquid crystals clearly have a potential for use in AFLC display devices since they exhibit an orientation-independent dark ground state if a horizontal surface-stabilized alignment can be 


\section{J. LAGERWALL ET AL.}

achieved. However, the requirements on the liquid crystal and its alignment are quite strict. The study of $(S, R)$ - M7BBM7 as OAFLC host shows that a number of problems not encountered with the previously used OAFLC mixtures may render some OAFLC materials useless in a device.

Most likely, the only way of achieving the required optical tilt of $\theta \approx 45^{\circ}$ over the whole working temperature range of the device is to use a material with a strongly first-order tilting transition since such a transition is often coupled to a large tilt and leaves little room for temperature dependence of the tilt angle. However, it may induce visible textural defects, giving rise to scattering and thereby ruining the excellent dark state, as observed in the host used in this study. For OAFLC devices - just as for any AFLC device - it is of vital importance that the $\mathrm{SmC}_{\mathrm{a}}{ }^{*}$ phase is stable throughout the temperature range in which the device is expected to be used. Equally important is that this phase, in its anticlinic state, is quickly reformed after removing the field used for switching the liquid crystal to its synclinic state during device operation. Since many AFLCs exhibiting a SmC* phase in the phase sequence have a tendency to stay in this phase when the cell gap is small, the presence of the $\mathrm{SmC}^{*}$ phase may pose a problem for AFLC device operation. In the chirally doped mixture studied here the $\mathrm{SmC}^{*}$ phase was supercooled to room temperature, thus actually expelling the desired $\mathrm{SmC}_{\mathrm{a}}{ }^{*}$ phase.

The fact that the previously studied partially fluorinated OAFLC mixtures do not suffer from problems with textural defects and supercooled SmC* phase shows that the materials development for OAFLCs so far is clearly on the right track. But as the helical pitch of these mixtures is still too short, it would be very interesting to mix one of these OAFLC mixtures with the $(S, R)$ - M7BBM7 compound used in the present study. Both components have $\theta \sim 45^{\circ}$ but the problems of one are not present in the other. At some mixing ratio, a highly useful OAFLC material might arise.

Acknowledgements

This work has been funded by the EU Research program SAMPA (Synclinic and Anticlinic Mesophases for Photonics Applications)

\section{References}

[1] K. D'havé, A. Dahlgren, P. Rudquist, J.P.F. Lagerwall, G. Andersson, M. Matuszczyk, S.T. Lagerwall, R. Dabrowski and W. Drzewinski, Ferroelectrics, 244, pp. 415-428 (2000) 
[2] K. D'havé, P. Rudquist, S.T. Lagerwall, H. Pauwels, W. Drzewinski and R. Dabrowski, Appl. Phys. Lett., 76, 24, pp. 3528-3530 (2000)

[3] S.T. Lagerwall, A. Dahlgren, P. Jägemalm, P. Rudquist, K. D'havé, H. Pauwels, R. Dabrowski and W. Drzewinski, Adv. Func. Mater., 11, 2, pp. 87 - 94 (2001)

[4] G. Scalia, P. Rudquist, D.S. Hermann, K. D'havé, J.R. Sambles and S.T. Lagerwall, J. Appl. Phys., 91, 12, pp. 9667 - 9675 (2002)

[5] P. Rudquist, J.P.F. Lagerwall, J.G. Meier, K. D'havé and S.T. Lagerwall, Phys. Rev. E., 66, 061708, pp. 061708 / 1 - 10 (2002)

[6] Y. Galerne, and L. Liebert, The Antiferroelectric Smectic O Liquid Crystal Phase, Oral O 27 presented at the Second symposium on ferroelectric liquid crystals, Göteborg, Sweden (FLC'89) , 1989

[7] Y. Galerne and L. Liebert, Phys. Rev. Lett., 64, 8, pp. 906-909 (1990)

[8] Y. Galerne and L. Liebert, Phys. Rev. Lett., 66, 22, pp. 2891-2894 (1991)

[9] P.E. Cladis and H.R. Brand, Liq. Cryst., 14, p. 1327 (1993)

[10] F. Giesselmann, A. Langhoff and P. Zugenmaier, Liq. Cryst., 23, 6, pp. 927-931 (1997)

[11] K. Miyasato, S. Abe, H. Takezoe and A. Fukuda, Jap. J. Appl. Phys. Lett., 22, p. L661 (1983)

[12] F. Giesselmann, J.P.F. Lagerwall, G. Andersson and M.D. Radcliffe, Phys. Rev. E., 66, 051704, pp. 051704 / 1-5 (2002)

[13] J.P.F. Lagerwall, F. Giesselmann and M.D. Radcliffe, Phys. Rev. E, 66, 031703, pp. 031703-1 - 031703-11 (2002)

[14] J.P.F. Lagerwall, D.D. Parghi, D. Krüerke, F. Gouda and P. Jägemalm, Liq. Cryst., 29, 2, pp. 163-178 (2002) 